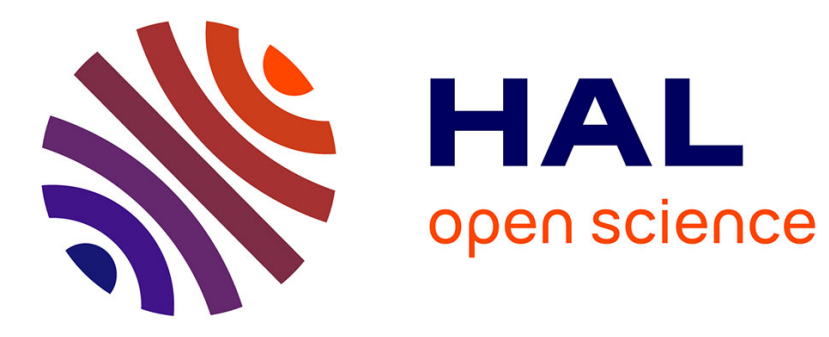

\title{
NP Navigator: A New Look at the Natural Product Chemical Space
}

Yuliana Zabolotna, Peter Ertl, Dragos Horvath, Fanny Bonachera, Gilles Marcou, Alexandre Varnek

\section{- To cite this version:}

Yuliana Zabolotna, Peter Ertl, Dragos Horvath, Fanny Bonachera, Gilles Marcou, et al.. NP Navigator: A New Look at the Natural Product Chemical Space. Molecular Informatics, 2021, 40 (9), pp.2100068. 10.1002/minf.202100068 . hal-03371813

\section{HAL Id: hal-03371813 \\ https://hal.science/hal-03371813}

Submitted on 12 Oct 2021

HAL is a multi-disciplinary open access archive for the deposit and dissemination of scientific research documents, whether they are published or not. The documents may come from teaching and research institutions in France or abroad, or from public or private research centers.
L'archive ouverte pluridisciplinaire HAL, est destinée au dépôt et à la diffusion de documents scientifiques de niveau recherche, publiés ou non, émanant des établissements d'enseignement et de recherche français ou étrangers, des laboratoires publics ou privés. 


\title{
NP Navigator: a New Look at the Natural Product Chemical Space
}

\author{
Yuliana Zabolotna ${ }^{a}$, Peter Ertl ${ }^{b}$, Dragos Horvath ${ }^{a}$, Fanny Bonachera ${ }^{a}$, Gilles Marcou ${ }^{a}$, Alexandre Varnek ${ }^{*}$ \\ [a] University of Strasbourg, Laboratoire de Chemoinformatique , 4, rue B. Pascal, Strasbourg 67081 (France) *e-mail: \\ varnek@unistra.fr \\ [b] Novartis Institutes for BioMedical Research, Novartis Campus, CH-4056, Basel, Switzerland
}

\begin{abstract}
Natural products (NPs), being evolutionary selected over millions of years to bind to biological macromolecules, remained an important source of inspiration for medicinal chemists even after the advent of efficient drug discovery technologies such as combinatorial chemistry and high-throughput screening. Thus, there is a strong demand for efficient and user-friendly computational tools that allow to analyze large libraries of NPs. In this context, we introduce NP Navigator - a freely available intuitive online tool for visualization and navigation through the chemical space of NPs and NP-like molecules. It is based on the hierarchical ensemble of generative topographic maps, featuring NPs from the COCONUT, bioactive compounds from ChEMBL and commercially available molecules from ZINC. NP Navigator allows to efficiently analyze different aspects of NPs - chemotype distribution, physicochemical properties, biological activity and commercial availability of NPs. The latter concerns not only purchasable NPs but also their close analogs that can be considered as synthetic mimetics of NPs or pseudo-NPs.
\end{abstract}

Keywords: chemoinformatics, natural products, chemical space, visualization, pseudo-NPs

\section{Introduction}

For centuries, natural products (NPs) were the only source of traditional medicines all over the world. Being evolutionary selected over millions of years to bind to biological macromolecules, they are able to selectively interact with many specific targets within the cell ${ }^{1}$. Therefore, NPs and their molecular frameworks remained an important source of inspiration for medicinal chemists even after the advent of efficient drug discovery technologies such as combinatorial chemistry $^{2,3}$ and high-throughput screening ${ }^{4,5}$. According to a comprehensive analysis, $6 \%$ of all smallmolecule drugs approved between 1981 and 2014 are unaltered NPs, 26\% are NP derivatives, and 32\% are NP mimetics and/or contain an NP pharmacophore ${ }^{6}$.

Over the past 20 years quite a large number of scientific reports exhaustively analyzed the chemical space of NPs in the medicinal chemistry context. Several studies were dedicated to the analysis of structural and physicochemical features of different libraries of $\mathrm{NPs}^{7,8}$ as well as their comparison to drugs and synthetic combinatorial libraries ${ }^{9-20}$. In addition, several models were proposed for distinguishing between natural products and synthetic molecules ${ }^{21-23}$. All of these reports contributed to a better understanding of NP-distinctive features, like heteroatom composition, number of rings, degree of saturation etc. In numerous publications, it was shown that NPs occupy parts of the chemical space not explored by available screening collections, which makes them valuable components of screening libraries used in drug discovery and increases the importance of computational tools for navigation of NP chemical space ${ }^{24}$.

Different methods are suitable for this task and a lot of them have been already used to analyze libraries of compounds of natural origin ${ }^{25}$. Principal component analysis (PCA) ${ }^{26}$ and scaffold trees ${ }^{27}$ were most often used, but self-organizing maps ${ }^{28}$ and generative topographic mapping (GTM) ${ }^{29}$ were also applied. Most of the numerous articles in this field simply report static results of particular compound library analysis, not allowing readers to explore the chemical space of NPs by themselves. To our best knowledge, there are only two web-based open platforms providing users with a certain level of interactivity and exploration freedom. The first one is an interactive web portal associated to The Natural Products Atlas - a database of microbial natural products that includes 24,594 compounds and associated data ${ }^{30}$. A similarity-based network is used to cluster and visualize these compounds providing the ability to browse and search through them. The second platform, D - Peptide Builder is a peptide generator, that also allows to visualize chemical space of peptides from different libraries using PCA and t-SNE plots ${ }^{31}$. However, both of them are limited to just a few distinct compound classes, visualizing only particular segments of the chemical space of NPs. Moreover, these database interfaces were not specifically designed for in-depth exploration, but rather for 
demonstrative purposes. For example, it is impossible to change "visualization perspective", i.e. display distribution of different properties that users may be interested in. D - Peptide Builder does not even allow to display chemical structures - only compound names appear on the plot. None of these platforms allow to project user-defined molecules for comparison with the database content.

In this context we present NP Navigator - a free, intuitive on-line tool for visualization and navigation through the chemical space of NPs and NP-like molecules. It is based on the hierarchical ensemble of generative topographic maps, featuring NPs from the COlleCtion of Open NatUral producTs (COCONUT) ${ }^{8}, 32$, bioactive compounds from $\mathrm{ChEMBL}$ and commercially available molecules from $\mathrm{ZINC}^{33}$. Being a nonlinear probabilistic dimensionality reduction method $^{34}$, GTM is well suited to power NP Navigator. It has already proven to be a successful approach for visualization and versatile analysis of large chemical libraries ${ }^{35-37}$. Hierarchical extension of GTM, combined with Maximum Common Substructure (MCS) detection ${ }^{36}$ allows to establish the link between the generalized visualization of the known chemical space of NPs/NP-like molecules and structural features of each separate compound.

As a result, NP Navigator allows to efficiently analyze different aspects of NPs - chemotype distribution, physicochemical properties, (reported and/or predicted) biological activity and commercial availability of NPs. The latter concerns not only purchasable NPs but also their close analogs that can be considered as pseudo-NPs ${ }^{38}$. Users are welcome not only to browse through hundreds of thousands of compounds from ZINC, ChEMBL and COCONUT but also project a small dataset of external molecules that play the role of "chemical trackers" allowing to trace particular chemotypes in the NP chemical space and detect analogs of the compound of interest.

Web-based implementation of NP Navigator is freely accessible at the link https://infochm.chimie.unistra.fr/npnav/chematlas userspace.

\section{Materials and Methods}

\section{Data preparation}

\section{Natural products}

The COCONUT database v. 2020.4 is a free and open collection of more than 426,000 -structures that were obtained by retrieving data from 53 sources and collecting additional data from the literature. However,

- $\quad$ molecules with NP-likeness score $22<-0.5$

- typical chemotypes privileged in synthetic compounds (polyhalogenated hydrocarbons, sulfonamides, thioureas etc.)

are not genuine NPs in our opinion, and were not considered in the present work.

The remaining 254,024 compounds have been standardized according to the procedure implemented on the virtual screening server of the Laboratory of Chemoinformatics at the University of Strasbourg (infochimie.ustrasbg.fr/webserv/VSEngine.html) using the ChemAxon Standardizer ${ }^{39}$. That included:

- dearomatization and final aromatization (heterocycles like pyridone were not aromatized);

- $\quad$ conversion to canonical SMILES;

- $\quad$ salts and mixture removal; neutralization of all species, except nitrogen (IV);

- the major tautomer generation

- $\quad$ stereochemical information removal.

Stereochemical information has been ignored due to the fact that ISIDA descriptors ${ }^{40}$, used in this work, would not capture it, anyway. As a result, 253,893 unique "stereochemistry-agnostic" molecular graphs remained. Each unique entry was linked to all the molecular IDs of the one or more stereoisomeric forms under which it actually appears in COCONUT.

\section{In-Stock commercially available compounds}

9,218,095 In-Stock compounds of "standard" reactivity have been downloaded from the ZINC20 website in October 2020. After standardization and duplicate deletion 6,460,596 compounds remained. Only 586,235 of them have NPlikeness scores higher than -0.5. These compounds (further - NP-like ZINC dataset) were used to define NP-Like commercially available chemical space. 


\section{Tangible commercially available compounds}

1.36 billion tangible compounds (not available for immediate purchase but might be synthesized upon request) were collected from the ZINC15 website in January 2019. After standardization, around 800 million stereochemistrydepleted tangible ZINC compounds remained, out of which $84,531,030$ tangible NP-like compounds passed the NPlikeness >-0.5 filter.

\section{Biologically tested compounds}

ChEMBL (version 26) ${ }^{41}$ served as a reference dataset for biologically tested molecules. 1,950,765 compounds have been collected in May 2020. After standardization, 1,721,155 unique compounds with known biological activities were kept.

The intersection of standardized ChEMBL and COCONUT returned 44,947 biologically tested NPs. Only 6,881 of them demonstrated dose-response activity on some target, with a activity value less than $10 \mu \mathrm{m}-$ active NPs. They were further classified with respect to their target family:

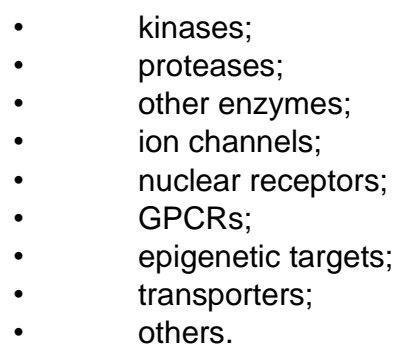

\subsection{Generative topographic mapping}

Generative topographic mapping (GTM) is a dimensionality reduction method originally described by Bishop ${ }^{34}$. The algorithm performs a non-linear projection from the initial $\mathrm{N}$-dimensional space into a $2 \mathrm{D}$ latent space. In chemoinformatics the former is defined by the $\mathrm{N}$-dimensional descriptor vectors assigned to each molecule of the dataset. The latent space resumes to a manifold, which is defined by a set of radial basis functions (RBF). The manifold is evaluated on sample points termed « nodes »At the training stage, the shape of the manifold is fitted to pass through the densest regions of the "frame set" (the pool of molecules used to probe the chemical space of interest). Then the nodes are folded back in 2D plane, as a squared grid.

By contrast to Self-Organizing Maps ${ }^{42}$, GTM assigns each molecule not to only one "winning" node but fuzzily distributes it over all nodes, with larger probabilities ("responsibilities") for near nodes. For each compound, responsibilities sum to one. Such a smooth projection supports the creation of GTM landscapes - 2D plots of cumulated compound responsibilities, colored by average values of different properties, e. g. density, biological activity, assigned class, etc. GTM landscapes can be used for chemical space analysis, library comparison or as a basis for building QSAR models ${ }^{43-46}$.

\subsection{Universal NP map: concept and construction}

Universal GTMs have been introduced by Sidorov et $\mathrm{al}^{47}$ and further developed by Casciuc et al ${ }^{48}$. They were defined as the "best compromise" maps, providing satisfactory predictive performance with respect to very diverse biological properties. Seven universal maps of the ChEMBL chemical space, defined by ISIDA fragment descriptors, have been "evolved" by a genetic algorithm (GA) ${ }^{49}$ in the map parameter space (including descriptor choice, grid size, manifold flexibility controls, etc, as key degrees of freedom). An average predictive performance over 236 biological activities was used as an objective function in a search for the best GTM parameters. These GTMs were proven to successfully serve as hosts for 618 (later extended to 749) activity landscapes associated with the respective targetspecific structure-activity ChEMBL compound series. Later they were combined in a consensus model implemented as an on-line GTM-based Profiler (http://infochim.u-strasbg.fr/webserv/VSEngine.html).

Unfortunately, due to the limited number of NPs in ChEMBL, their applicability to NP chemical space analysis is not appropriate. A dedicated NP map was evolved as part of this work, albeit with a different, Pareto-front driven multiobjective strategy. A fixed frame set 16,025 randomly selected NPs was used. The maps were challenged to maximize: 
(a) the pairwise separation of NPs assigned to different activity classes (vide supra): for each of the $9^{*}(9-1) / 2=36$ pairs $\left(C_{i}, C_{j}\right)$ of distinct activity classes. The mutual separation of respective class members on the landscape is reported as a cross-validated balanced accuracy (BA) score and used as an objective function for best GTM parameters selection. Maps in which the compound sets significantly overlap will witness members of class $C_{i}$ projecting amid a cluster of representatives of $\mathrm{C}_{\mathrm{j}}$ during cross-validation, resulting in lower BA. By contrast, parameter choices defining maps in which members of $C_{i}$ and $C_{j}$ are projected on distinct areas of the manifold would not lead to such mispredictions and thus higher BA values will be obtained.

(b) the Shannon entropy of a large (24K) random subset of NPs, normalized with respect to the maximal entropy achievable on a map of $\mathrm{N}$ nodes. Recall that the Shannon entropy of a mapped compound library is $S=-\sum_{i=1}^{N} f_{i} \ln f_{i}$, where $\mathrm{f}_{\mathrm{i}}$ is the fraction of "compounds residing in node $\mathrm{i}$ " in terms of cumulated responsibilities (cumulated responsibility of node i by compound library size L). The "ideal" maximal entropy map providing the most homogeneous possible mapping would equally split the library over all its nodes, thus $f_{i}=1 / N$ and $S_{\text {max }}=$ $-\sum_{i=1}^{N} \frac{1}{N} \ln \frac{1}{N}=\ln N$.

The entropy objective, equaling $S / \ln (\mathrm{N})$ becomes independent of map size and characterizes the homogeneity of the NP distribution over the landscape.

Unlike in the previous universal map strategy - where the initial 236 balanced accuracy objectives were "collapsed" into a single fitness score (their plain arithmetic average minus standard deviation) the present approach considered the above $36(\mathrm{BAs})+1(\mathrm{~S} / \mathrm{lnN})$ as independent objectives, and the Pareto front of non-dominated maps was considered as the current "breeding" population. A new "individual" obtained by standard genetic operators is evaluated by generating the map according to the parameter values encoded in its chromosome, required compounds are projected on it and the 37 objective scores are estimated. If another, previously discovered parameter configuration is known to have produced a map which is better than the "new born" one with respect to each of the 37 objectives, the newborn configuration is "dominated" and will be discarded. Otherwise, the configuration is better than the so-far found with respect to at least some of these objectives and is allowed to enter the current population.

\section{Hierarchical GTM (HGTM)}

While analyzing hundreds of thousands of compounds, map resolution may be insufficient for meaningful chemotype clustering. In such a case, a hierarchical zooming approach is required to improve class separation on the finer scale of zoomed maps. Hierarchical GTM (HGTM), a.k.a "Zooming"50 is a technique that trains a new map on a set of compounds extracted from a given zone on the parent map, in order to further resolve compound clusters with degenerated responsibility patterns. This approach, combined with a maximum common substructure (MCS) detecting algorithm was previously implemented in AutoZoom ${ }^{36}$ - an in-house tool that has been developed for the chemotypes identification in the heavily populated zones of the map. First, it separates the map into small zones (3x3 nodes) and detecting "overcrowded" zones (of more than 1000 compounds). In this work, zone "residents" were counted as compounds for which the sum of responsibilities over the nodes in the particular zone is higher than 0.85 . A pool of $10 \%$ of residents (but not less than 1000) were selected using the dissimilarity principle and used as a frame set for the new GTM manifold construction (with map parameters "borrowed" from the parent map). Successive zooming of all overcrowded zones was hierarchically performed until all are eventually broken up into clusters of less than 1000 compounds and then submitted to the MCS extraction, realized using ChemAxon's JChem engine ${ }^{39}$. Only MCS covering at least $30 \%$ of each of the molecules were reported. After the primary identification of the specific MCS, they were submitted as substructure search queries in order to verify whether they are genuinely absent from the entire subspace (and not only from the zones targeted by successive zooming)

\section{RESULTS AND DISCUSSION}

\section{Optimal NP-Umap}

By definition, a Pareto-front driven optimization does not produce a single best solution unless all objectives are correlated and a configuration simultaneously maximizing all of them exists. This is not expected to be the case here. Thousands of map configurations were retrieved, each having locally some competitive edge over others, in terms of specific objectives. Note that perfect separation of the members of considered classes is neither necessary nor expected (actually, some compounds are "promiscuous" and included in several classes - ion channels and GPCRs, for example, are notoriously sharing many actives). In these cases, the same molecule is present twice in the cross- 
validation set - labeled both as " $\mathrm{C}_{\mathrm{i}}$ " and " $\mathrm{C}_{\mathrm{j}}$ ", making overlap unavoidable. The goal is to maximize separation in as far as this is possible, not to aim for perfect separation.

Eventually, one map was hand-picked, amongst those with worst balanced accuracy exceeding some minimal threshold (here, 0.59), all while being based on the technically most convenient descriptors amongst the ones allowing such level of performance. The selected "best" map consists of 1,225 nodes (35×35) coupled with 324 RBFs (18x18). The descriptors used to define NPs chemical space are ISIDA symmetrical atom-centered fragments with topological distance from 1 to 2 including both atoms and bonds information. These are easier to calculate than the topological pharmacophore fragments very often encountered in good maps (the latter require an additional pharmacophore typing step, which may be expensive as it involves an explicit protonation state prediction). The average BA in class separation is 0.67 .

\section{Chemical space of natural products - chemotype distribution}

The entire NP dataset has been projected onto the newly constructed NP-Umap. Figure 1 shows the obtained density landscape. Multicolored areas correspond to the highly populated regions, while gray color defines moderately occupied areas. White zones are empty. Several regions of the high density correspond to some of the most common NP families e.g. lipids, alkaloids, sugars, flavonoids etc.

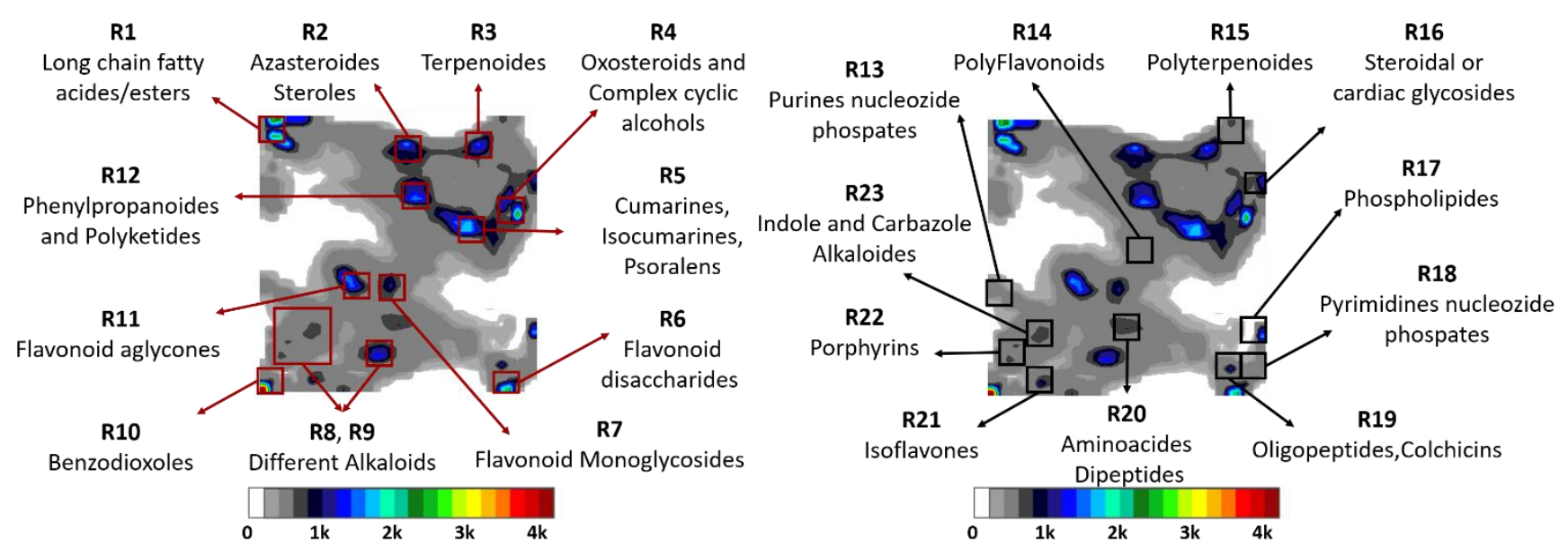

Figure 1. Density landscape of NPs from COCONUT. On the left - chemotypes for the highly populated regions, on the right - for the low populated ones. Multicolored areas correspond to the highly populated regions, while gray color defines moderately occupied areas. White zones are empty.

In general, the northern part of the map corresponds to the NPs with a high proportion of carbon atoms - longchain fatty acids and corresponding lipids (R1), steroid-like compounds (R2), terpenoids (R3) etc. While heading south-east, the number of oxygen atoms increases resulting in dense regions of polyketides (R12), oxosteroids (R4) coumarins and psoralenes (R5). Close to the oxosteroids, a small island of steroidal or cardiac glycosides (R16) can be found - compounds that contain both carbocyclic steroid moiety and oxygen-enriched sugar fragments. In the central part, flavone-containing compounds can be found - polyflavonoids (R14), flavonoid aglycones (R11) and monoglycosides (R7). However, flavonoid disaccharides are residing on the far south-east of the map (R6), next to the colchicines and oligopeptides (R19). At the same time, aminoacids and dipeptides (R20) are neighboring flavonoid monoglycosides from one side and large area of N-heterocycles - different types of alkaloids (R8, R9) from another. Extreme south-west of the map is populated by numerous benzodioxol-containing compounds and their analogs.

Interestingly, nucleotides are not situated in the same regions - pyrimidine nucleoside phosphates (R18) reside close to phospholipids (R17) on the south-eastern part of the map, while purine nucleoside phosphates (R13) are found in the far west - neighboring the alkaloids area. Such distancing of (by human perception) similar compound subfamilies illustrates the competitive contribution of several underlying chemotypes to the compound's position in 
the chemical space. Pyrimidine nucleotides with their relatively smaller N-heterocycle moiety tend to be closer to phospholipids. In purines, N-heterocycles are dominant placing those compounds near the alkaloids area.

The NP-Umap supports a significant separation of the most common NP compound families, which makes it an efficient tool for NPs chemical space navigation. However, for more detailed structural analysis hierarchical zooming needs to be applied. In Figure 2, zooming of the alkaloid-containing region (R9) is shown as an example. With a better resolution, we can distinguish several density picks, corresponding to the different alkaloid subfamilies piperazine and piperidine containing sesquiterpene lactones, guanidine-containing alkaloids, indoline, indole, isoquinoline and rhazinilam alkaloids. While all are members of one of the largest NP classes and thus to some extend similar, they nevertheless possess unique structural features that could be captured only with a help of HGTM.

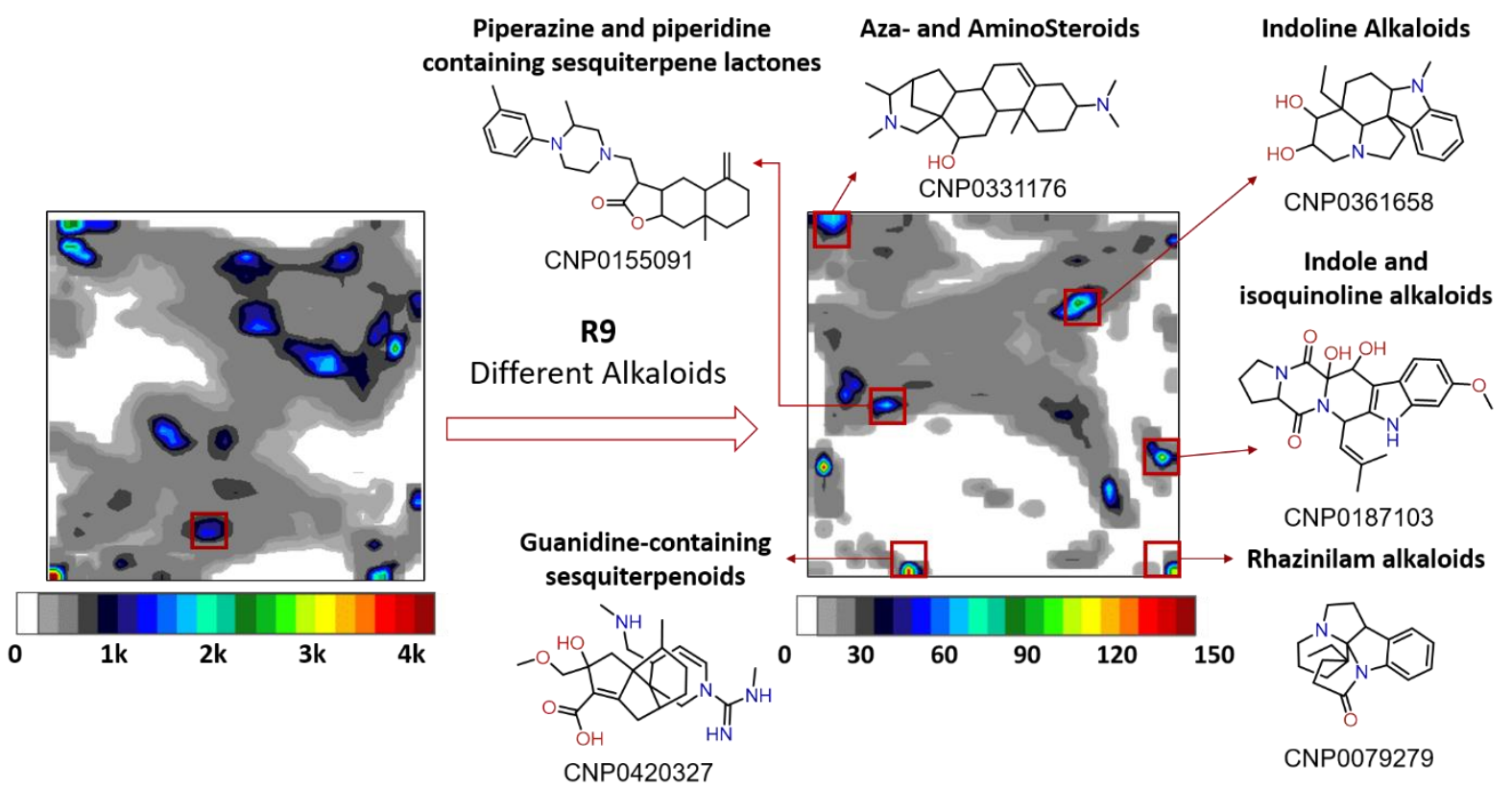

Figure 2. Zoomed density landscape for the region R9 that contains different type on alkaloids. On the finer scale of the zoomed map one can observe better chemotypes separation. Multicolored areas correspond to the highly populated regions, while gray color defines moderately occupied areas. White zones are empty.

\section{Commercial availability of natural products and amount of associated biological testing data, as functions of drug-likeness}

As already mentioned, multiple different landscapes can be created for a same map. They can be used separately or combined allowing to analyze projected compound libraries from different perspectives - comparing, for example, the availability of bioactivity test results versus commercial availability of NPs. COCONUT was intersected with ChEMBL and NP-like ZINC datasets, resulting in almost $45 \mathrm{~K}$ of biologically tested compounds and $11 \mathrm{~K}$ commercially available NPs, respectively. Their distribution within the entire COCONUT NP dataset is shown in Figure 3. The left-hand map is a fuzzy class landscape contrasting biologically untested NPs (COCONUT - ChEMBL) in black, versus experimentally tested NPs (COCONUTAChEMBL) in red, mixed regions in intermediate colors. On the right-hand map, commercially unavailable NPs (COCNUT - ZINC) - black regions - cover largely the same map zones as untested NPs (COCONUT-ChEMBL). It is no surprise that compounds that are difficult to access are not amongst the most tested ones. The middle map shows the COCONUT drug-likeness landscape, based on the drug-likeness (QED) score ${ }^{51}$. It varies from zero to one - the bigger the score the more drug-like properties the compound possesses. It appears that both biologically tested and commercially available NPs-enriched regions coincide fairly well with areas of the high QED values, showing that one of the driving forces of the NPs exploration in bioactivity and purchasability context is their physicochemical properties and thus their potential to be used as drugs. This is just one of many possible examples of how integrated analysis of multiple property landscapes can shed the light onto different aspects of the NPs chemical space providing generalized understanding of its global features. 


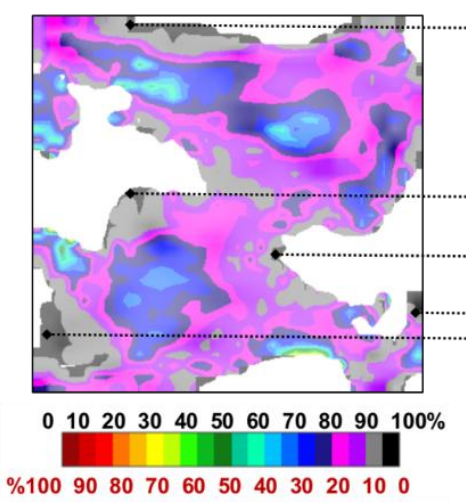

Not tested NPs - 209K cmpnds

Biologically tested NPs- $45 \mathrm{~K}$ cmpnds

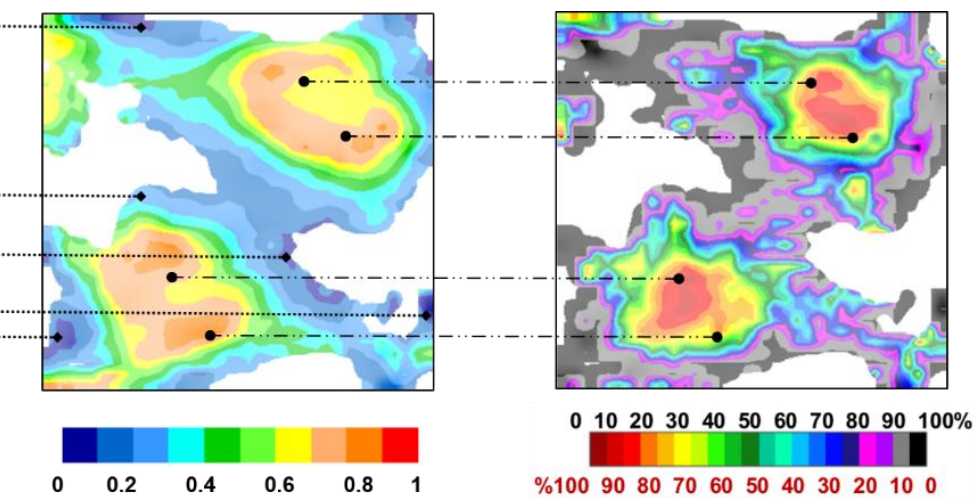

QED $=0$ - all properties are unfavourable QED=1 - all properties are favourable
Not available NPs $-243 \mathrm{~K}$ cmpnds Commercially available NPs- $11 \mathrm{~K}$ cmpnds

Figure 3. Amount of existing (ChEMBL-reported) NP bioactivity data and NP commercial availability relate to the druglikeness of compounds. Map on the left - class landscape comparing biologically tested (red) and not tested (black) NPs. Map in the middle - property landscape showing distribution of quantitative estimate of drug -likeness (QED) of NPs. Blue regions correspond to the compounds with all physicochemical parameters being unfavorable for oral drugs, red ones with all properties being favorable. Map on the right - normalized class landscape comparing commercially available (red) and not available (black) NPs.

\section{Natural Products vs NP-like ZINC compounds}

The newly constructed NP-Umap is not limited only to NPs - any compounds populating the regions of the chemical space, covered by the map can be projected. Considering the neighborhood behavior principle ${ }^{52}$, those compounds should be structurally similar to the natural compounds used for GTM construction - NP-like compounds - and thus possess similar properties. Mapping the external dataset of the NP-like commercially available compounds and their structural comparison with NPs can provide valuable insight into similarities and differences between artificially synthesized and naturally produced molecules. Reversely, pseudo-NP (synthetic analogs of natural compounds) detection of NP-zone residents stemming from synthetic sources can be easily performed.

Thus, 254k NPs and 586k NP-like ZINC compounds were projected onto NP-Umap. In Figure 4 the first map is a fuzzy class landscape where black regions correspond to the NPs and red - to the NP-like ZINC compounds. Even on the global "bird's-eye" scale of NP-Umap, regions significantly dominated by members of each library can be spotted. However, there are plenty of mixed zones, containing both NPs and commercially available NP-like compounds. In Figure 4, one example of the more detailed HTGM-based analysis is pursued. A mixed green zone (square of $3^{\star} 3$ nodes), containing 7902 compounds with almost 50:50 ratio of members of each library, has been zoomed resulting in a new map of finer scale with a better class separation - multiple regions occupied by compounds from only one library can be found. For further structural analysis of those regions, maximum common substructures (MCS) were used as a way to generalize structural features of compounds populating them. MCS was preferred over the popular scaffold concept due to its flexibility and adaptability. MCS can either contain only rings and linkers, in such a way coinciding with the corresponding scaffold or be more specific by including side-chain substituents if that is beneficial for capturing distinctive structural features of the analyzed libraries. Here we aimed to identify unique MCSs, found either only in COCONUT, or in NP-like ZINC respectively.

As a result of the iterative HGTM application, 241 HGTMs have been built with up to two levels of zooming. With the help of those maps 15,891 locally NP-like ZINC-specific MCSs and 9,357 locally COCONUT-specific MCS have been found. "Locally specific" means that in the analyzed region this MCS occurs only in one library. However, as observed with the nucleotides, compounds sharing similar structural patterns can be situated far from each other on the map due to the contribution of other underlying chemotypes to the molecule position. As a result, locally specific MCSs may still be present in the other library, but outside the analyzed area. Therefore, an additional substructure search is needed to ensure (absolute) specificity of locally identified MCSs. NP-Like ZINC-specific MCSs have been checked against COCONUT NPs leaving only 12,981 ZINC-specific MCSs (10,545 of which are absent also in the uncleaned COCONUT dataset). Local NP-specific MCSs in their turn have been substructure-queried against the NPlike In-Stock ZINC library, with 8,282 MCSs returning no matches. However, 1,337 of these NP-specific MCSs have been found in the NP-like Tangible ZINC dataset, making compounds incarnating them purchasable in principle 
(acquisition success rate for tangible compounds is around 70\%). The complete list of detected NP- and NP-Llke ZINC-specific chemotypes is available upon quick registration by the link https://forms.gle/LHQPvqitKEJv7e4K8.

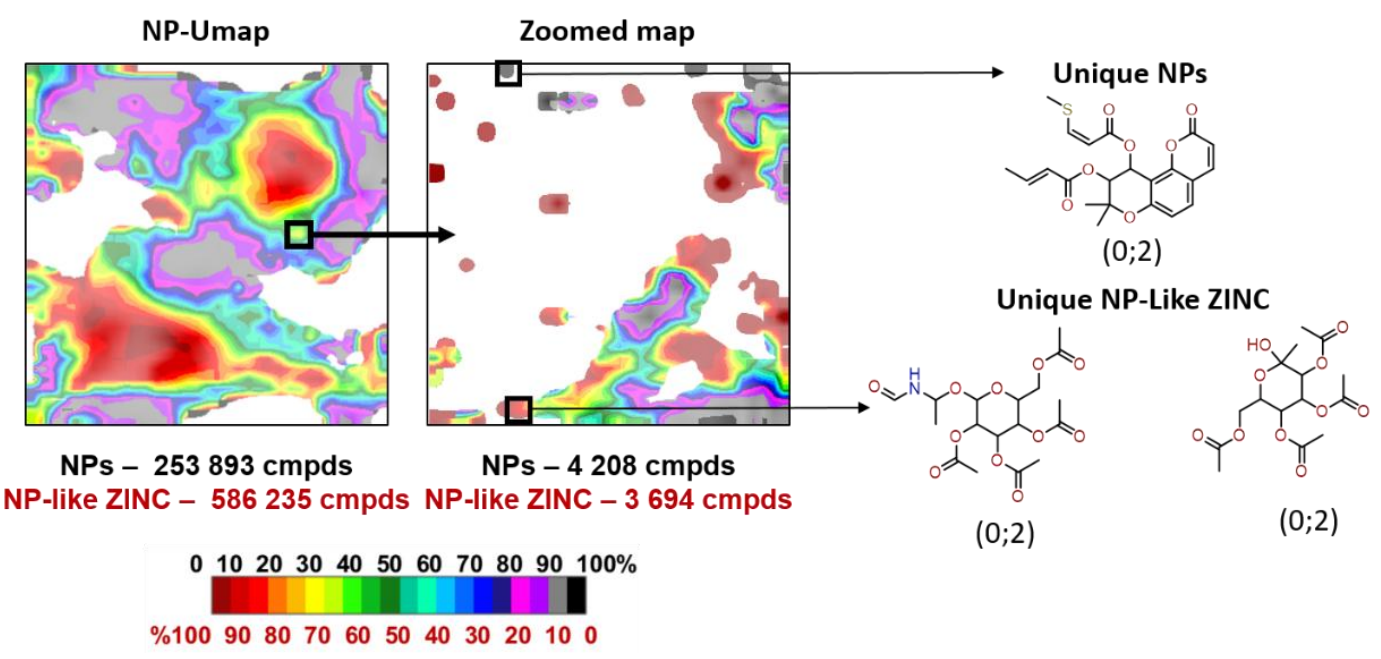

Figure 4. Examples of the zooming (HGTM) procedure in a search for NP-specific and ZINC-specific MCSs. First number in parenthesis gives number of hits in COCONUT, second one - in NP-like ZINC.

Figure 5 displays the most often encountered NP-like ZINC-specific and COCONUT-specific MCSs. The first number in parenthesis represents occurrences in COCONUT, the second in NP-like ZINC. Among the ZINC-specific MCSs there are some purely synthetic chemotypes like bicyclo(1.1.1)pentane derivatives (R4) or dioxaborolanes (R3). However, some contain typical rings often seen in NPs e.g. furane (R5) or pyrrole (R17). Here, the ring substitution patterns typically produced by chemical synthesis are conferring ZINC-specificity to these MCS. There are also ZINC-specific MCSs representing synthetic peptidomimetics (R10) and synthetically modified natural compounds (R6). In any case, $90 \%$ of them contain nitrogens as key heteroatoms. In contrast, the majority of COCONUT-specific MCSs corresponds to the complex carbo- or oxoheterocycles with oxygen-containing sidechains. Thus, nitrogen-containing compounds and alkaloids, in particular, are better explored by synthetic chemistry than complex oxygen-containing NPs.

\section{Biological activity of natural products}

As mentioned before, ChEMBL bioactivity data are available for about 45k NPs. Those compounds are almost evenly distributed around the map, typically within high QED regions (Figure 3). By contrast, the most common chemotypes for untested NPs (Figure 6) contain either complex ring systems or long hydrocarbon chains, shifting them outside of the drug-likeness domain.

NP-Umap can be also used for the target-based bioactivity analysis. Figure 7 and Figure 8 display fuzzy classification landscapes contrasting NP ligands of each of the target classes (C) used for NP-Umap optimization black regions - against NPs active against all other targets reunited into one non-C class - red zones. Note - non-C pool does not include any of COCONUT compounds that were not labeled by activity class. Landscapes have been normalized due to the high dataset imbalance (mid-range color green corresponds to zones populated by classes $\mathrm{C}$ and non-C at local cumulated responsibility ratio equaling the default ratio of those set sizes). Target class-specific MCSs are shown below, except for the 70 enzyme-specific MCSs out of which only 5 most populated are shown. 

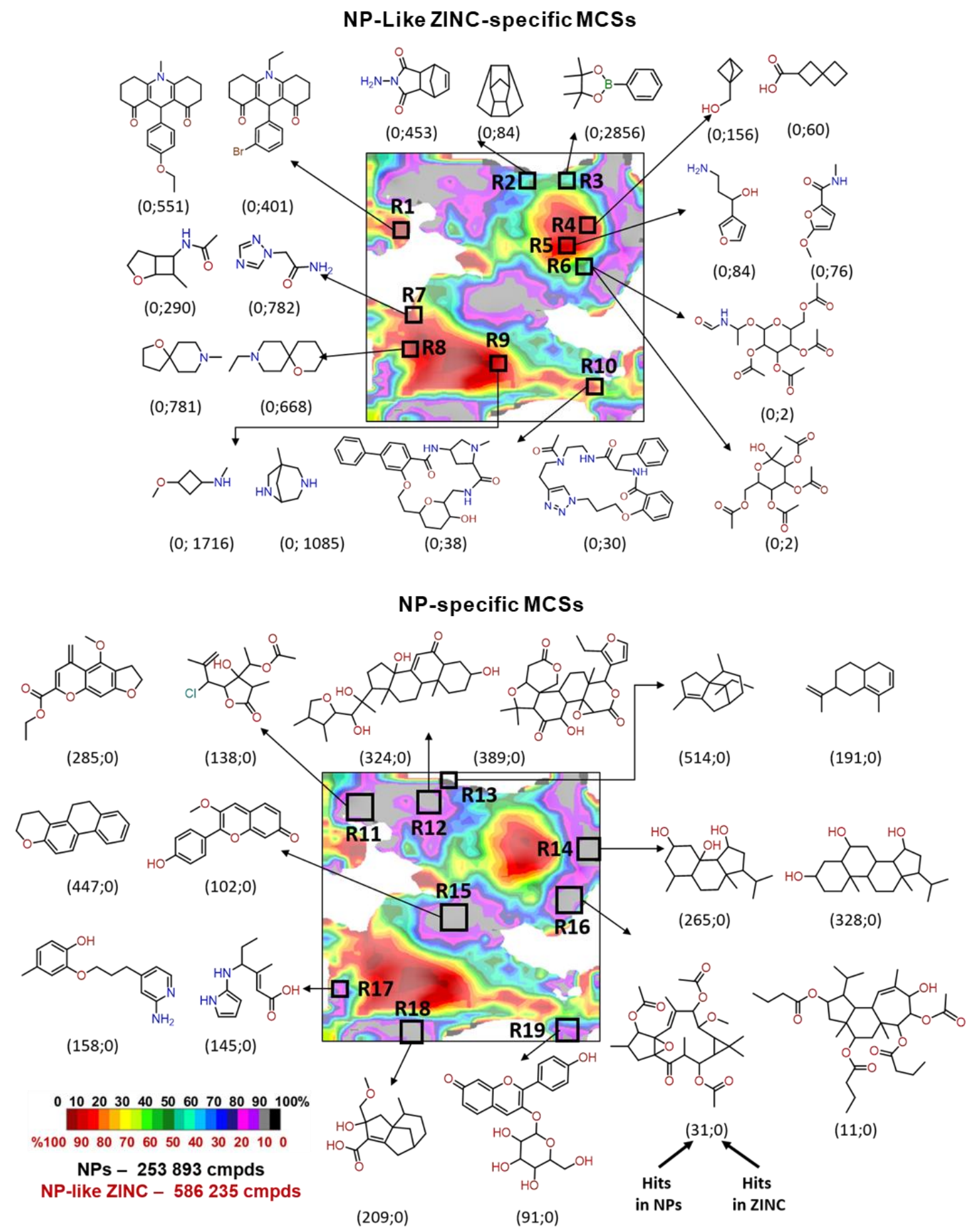

Figure 5. Class landscape comparing COCONUT natural products(black) with NP-like ZINC compounds(red). Upper scheme provides examples of ZINC-specific MCSs, while lower one demonstrates NP-specific MCSs. First number in parenthesis gives number of hits in c-COCONUT, second one - in NP-like ZINC. 


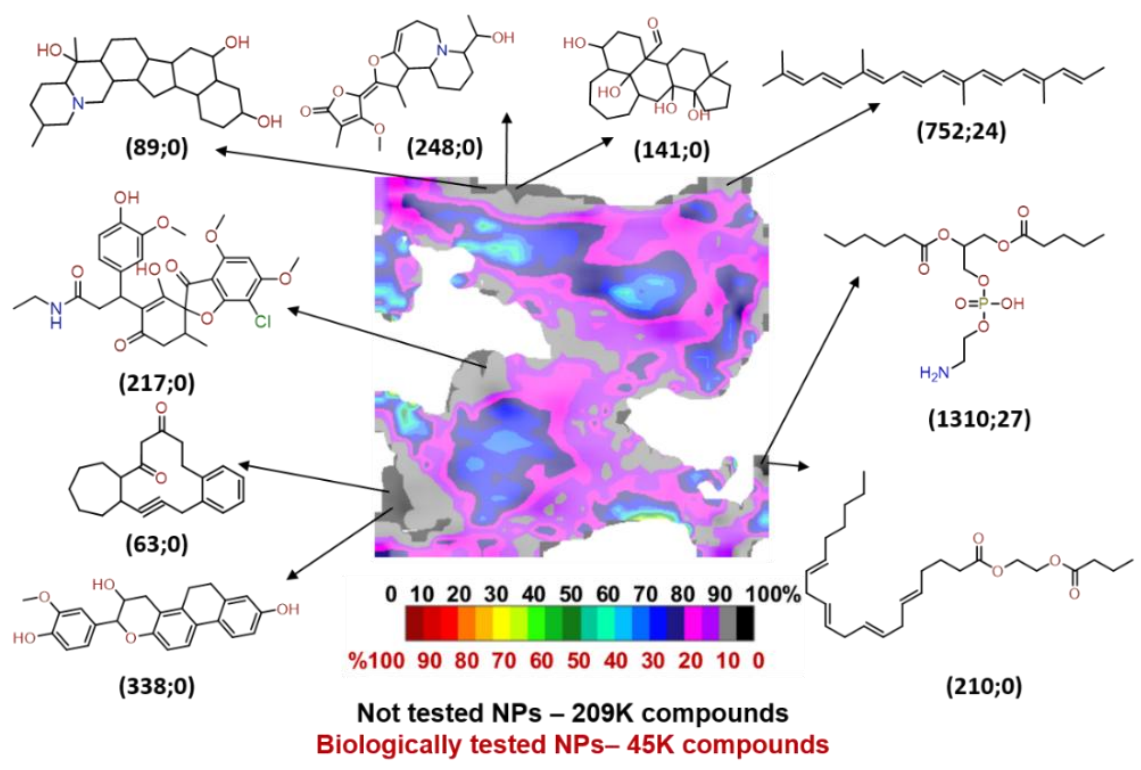

Figure 6. Class landscape comparing biologically tested (red) and not tested (black) NPs. Given substructures correspond to the MCSs, specific to the not tested subset. First number in parenthesis gives number of hits in not tested subset, second one - in tested.
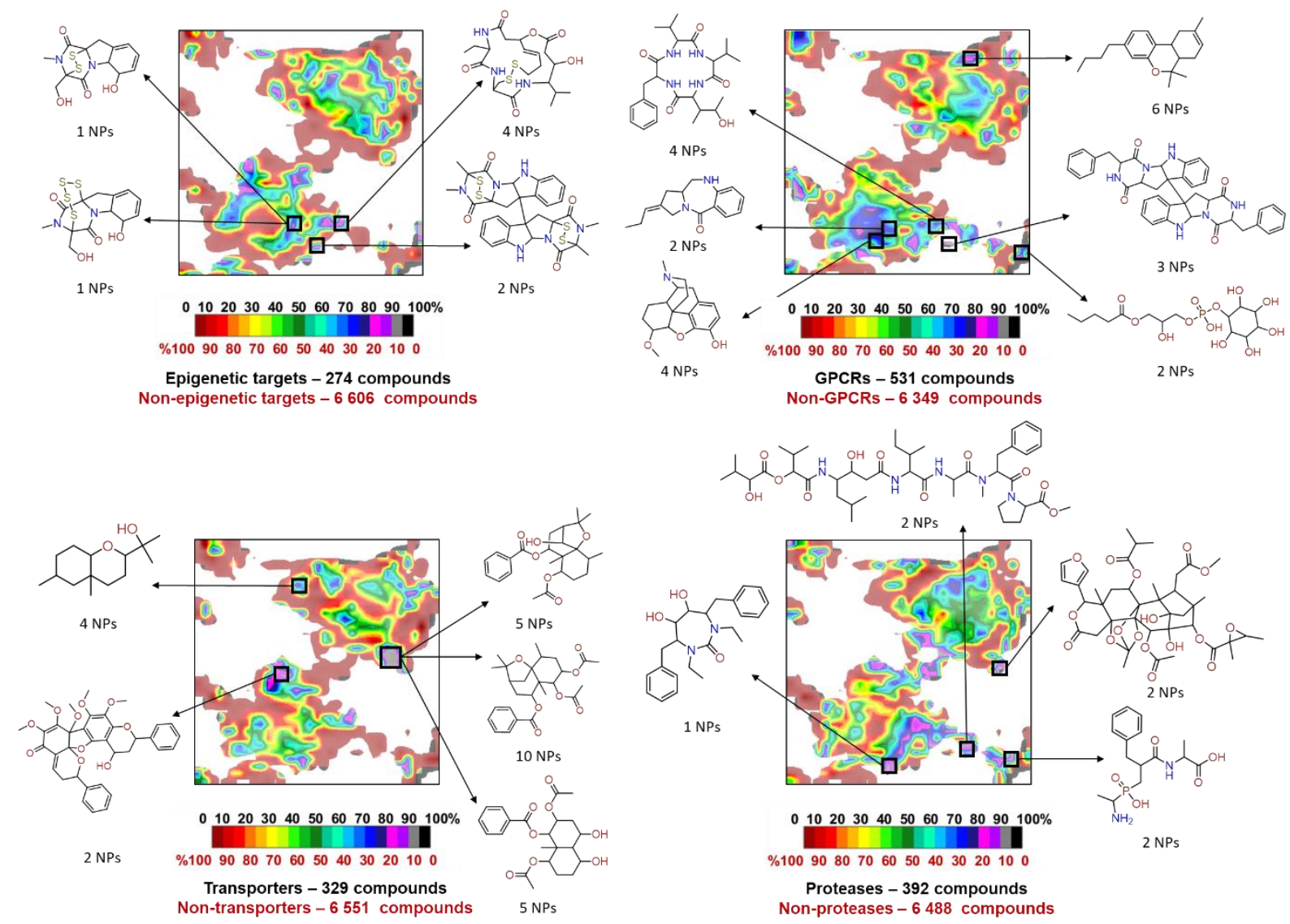

Figure 7. Target-specific NP chemotypes and corresponding regions of chemical space: epigenetic targets, GPCRs, transporters and proteases. 

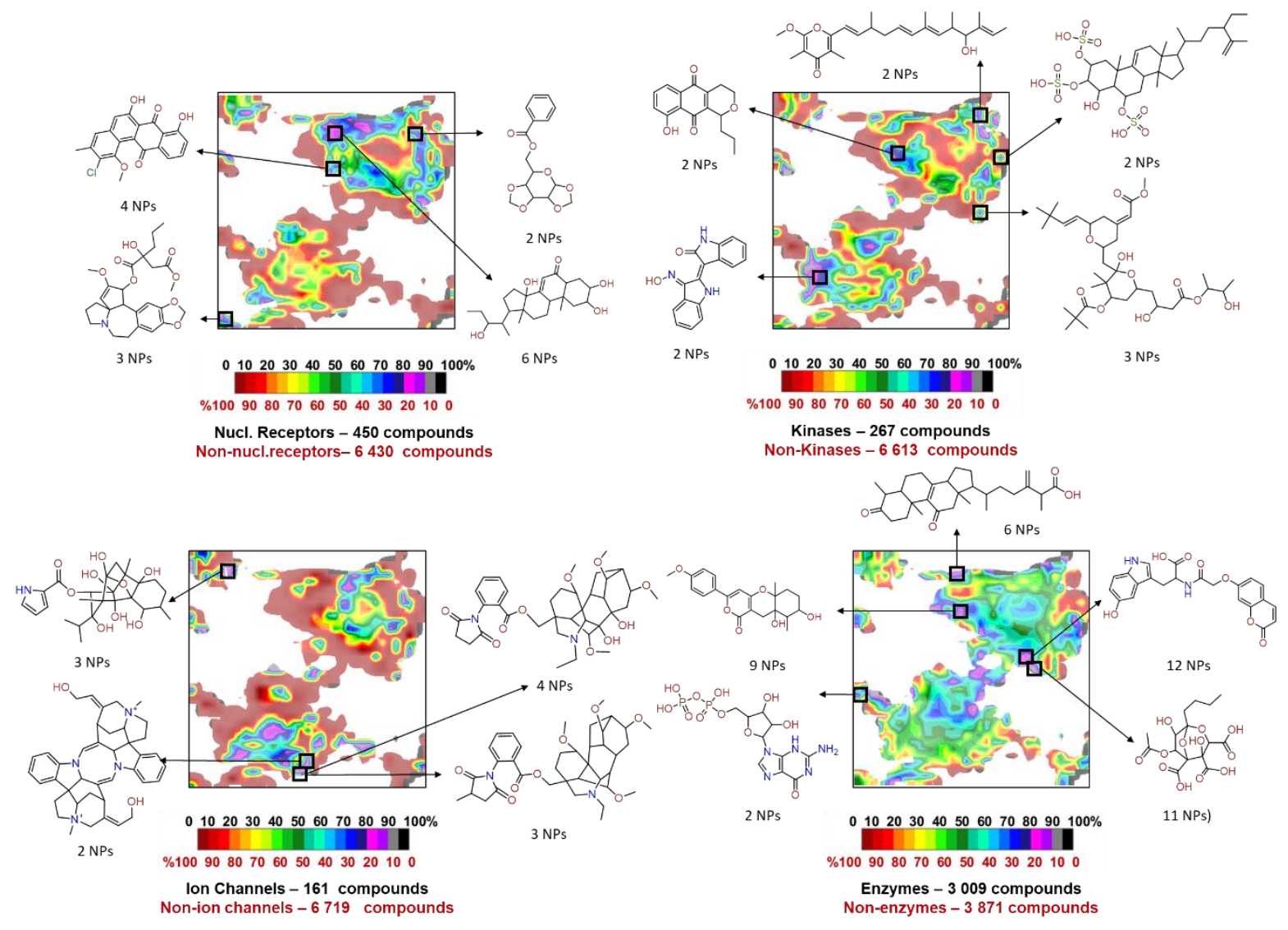

Figure 8. Target-specific NP chemotypes and corresponding regions of chemical space: nuclear receptors, kinases, ion channels and other enzymes

\section{NP Navigator}

The hierarchical ensemble of maps was used as the basis for NP Navigator - a multifunctional tool for the analysis of the chemical space of NPs and NP-like molecules. It is openly accessible via web-interface by the https://infochm.chimie.unistra.fr/npnav/chematlas userspace. NP Navigator provides access to the library of multiple pregenerated property landscapes - density, various physico-chemical parameters, QED, ZINC vs NPs and ChEMBL vs NPs comparative landscapes, biological activity landscapes, etc. Each predefined zone (square of $3^{\star} 3$ nodes) of these maps is assigned to the NPs, NP-like ZINC and ChEMBL compounds populating it. Those compounds as well as MCSs characterizing them can be displayed and/or downloaded. If the zone was zoomed, the HGTM landscape will be shown prior to the associated compounds list. In such a way users can by themselves navigate through the chemical space of NPs and explore its different aspects. NP Navigator can be used for different purposes - chemical space analysis, NP-like libraries comparison (Figure 4 and Figure 5), searching for the NP-analogs of the compound of interest (Figure 9), analysis of the biological activity of NPs (Figure 7 - Figure 9). 


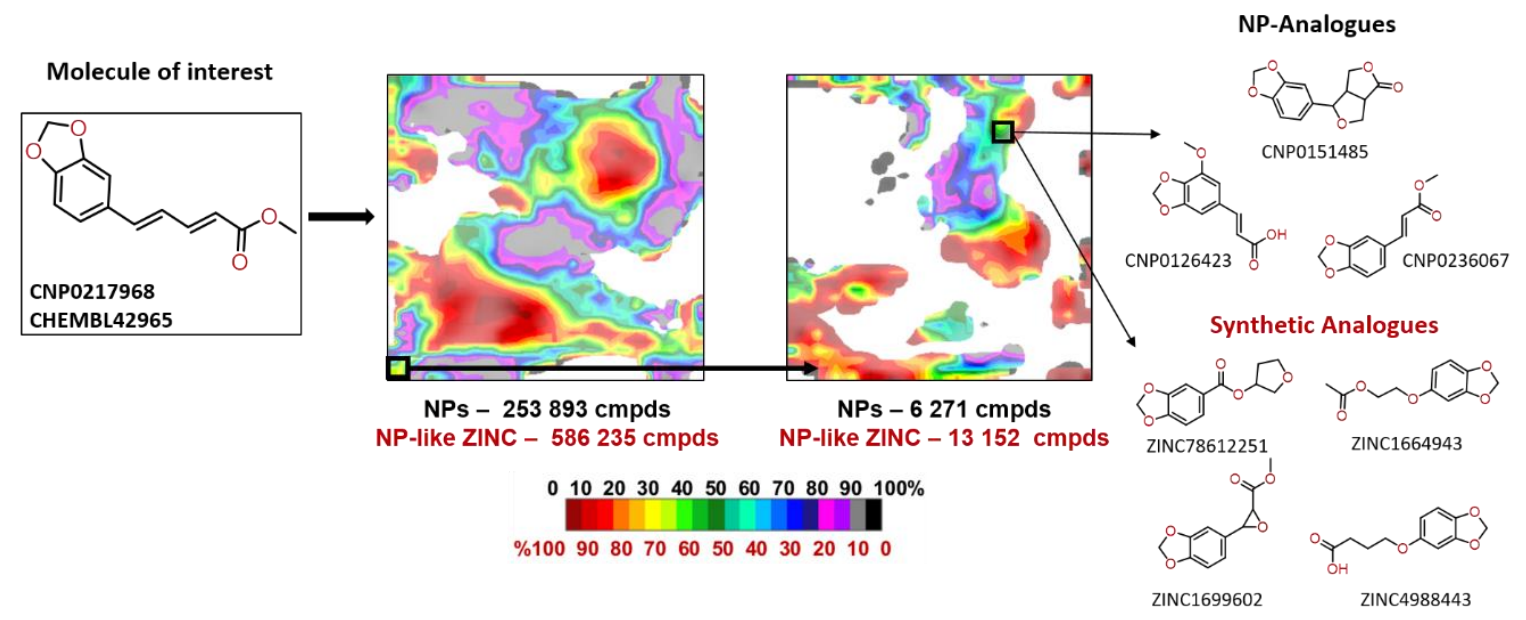

Figure 9. Search of the NPs and synthetic analogs of a compounds of interest using NP Navigator (241 GTM in total). After being projected onto the NP-Umap, compound is followed down to the last level of zoom. Neighboring compounds on the last zoomed map can be considered as a close NP-analogs and synthetic analogues of the initial compound of interest

\section{CONCLUSIONS}

In this work, hierarchical GTM has been used to perform a thorough analysis of the chemical space represented by natural products. More than 200 HGTMs based on the universal map of natural products (NP-Umap) have been constructed. It has been shown that the ensemble of those maps - accessible via web-interface NP Navigator provides a meaningful chemotypes separation, which can be used for structural analysis of NPs and in a search of natural or synthetic analogs of the molecule of interest.

Comparison of COCONUT NPs and NP-like ZINC subsets resulted in almost 20 thousand unique MCSs, specific to only one library (https://forms.gle/LHQPvaitKEJv7e4K8). $90 \%$ of ZINC-specific MCSs contain a nitrogen atom. Concerning NPs-specific MCSs, the majority of them correspond to the complex carbo- or oxoheterocycles with oxygen-containing sidechains. This illustrates the well-known fact that nitrogen-containing compounds in general and alkaloids, in particular, are better explored by synthetic chemistry than complex oxygen-containing NPs. ZINCspecific MCSs, being the chemotypes found in NP-like ZINC but never occurring in NPs, can be used as a filtering set applicable together with NP-likeness score in order to improve NP-likeness of the designed library.

Biological activity and purchasability of NPs have been also investigated. It was shown that one of the driving forces of NP-focused investigation for biomedical applications is their physicochemical profile and thus their potential to be used as drugs - NPs with a higher QED score tend to appear more often in ChEMBL and ZINC than other compounds.

NPs active against popular target families (kinases, proteases, other enzymes, ion channels, nuclear receptors, GPCRs, epigenetic targets, transporters), have been analyzed in order to find characteristic structural features unique for each of the ligand series. However, it appears, that NP active against different target classes may significantly overlap in the chemical space if those targets are naturally "promiscuous" with respect to each other's ligands. Thus only a few specific MCSs have been found for each target-based subset.

\section{REFERENCES}

1. Dixon, N.; Wong, L. S.; Geerlings, T. H.; Micklefield, J. Cellular targets of natural products. Nat. Prod. Rep. 2007, 24, 1288-1310.

2. Liu, R.; Li, X.; Lam, K. S. Combinatorial chemistry in drug discovery. Curr. Opin. Chem. Biol. 2017, 38, 117126.

3. Ramström, O.; Lehn, J.-M. Drug discovery by dynamic combinatorial libraries. Nature Reviews Drug Discovery 2002, 1, 26-36.

4. Inglese, J.; Auld, D. S. High Throughput Screening (HTS) Techniques: Applications in Chemical Biology. Wiley Encyclopedia of Chemical Biology 2008, 1-15. 
5. Macarron, R.; Banks, M. N.; Bojanic, D.; Burns, D. J.; Cirovic, D. A.; Garyantes, T.; Green, D. V. S.; Hertzberg, R. P.; Janzen, W. P.; Paslay, J. W.; Schopfer, U.; Sittampalam, G. S. Impact of high-throughput screening in biomedical research. Nature Reviews Drug Discovery 2011, 10, 188-195.

6. Newman, D. J.; Cragg, G. M. Natural Products as Sources of New Drugs from 1981 to 2014. J. Nat. Prod. 2016, 79, 629-661.

7. $\quad$ Chen, Y.; de Bruyn Kops, C.; Kirchmair, J. Data Resources for the Computer-Guided Discovery of Bioactive Natural Products. J. Chem. Inf. Model. 2017, 57, 2099-2111.

8. Sorokina, M.; Steinbeck, C. Review on natural products databases: where to find data in 2020. J. Cheminformatics 2020, 12, 20.

9. Wetzel, S.; Schuffenhauer, A.; Roggo, S.; Ertl, P.; Waldmann, H. Cheminformatic Analysis of Natural Products and their Chemical Space. CHIMIA 2007, 61, 355-360.

10. Stratton, C. F.; Newman, D. J.; Tan, D. S. Cheminformatic comparison of approved drugs from natural product versus synthetic origins. Bioorg Med Chem Lett 2015, 25, 4802-4807.

11. Wenderski, T. A.; Stratton, C. F.; Bauer, R. A.; Kopp, F.; Tan, D. S. Principal component analysis as a tool for library design: a case study investigating natural products, brand-name drugs, natural product-like libraries, and drug-like libraries. Methods Mol Biol 2015, 1263, 225-242.

12. Singh, N.; Guha, R.; Giulianotti, M. A.; Pinilla, C.; Houghten, R. A.; Medina-Franco, J. L. Chemoinformatic analysis of combinatorial libraries, drugs, natural products, and molecular libraries small molecule repository. $J$. Chem. Inf. Model. 2009, 49, 1010-1024.

13. Ertl, P.; Schuffenhauer, A. Cheminformatics analysis of natural products: Lessons from nature inspiring the design of new drugs. In Natural Compounds as Drugs: Volume II, Petersen, F.; Amstutz, R., Eds. Birkhäuser Basel: Basel, 2008; pp 217-235.

14. Rosén, J.; Gottfries, J.; Muresan, S.; Backlund, A.; Oprea, T. I. Novel Chemical Space Exploration via Natural Products. J. Med. Chem. 2009, 52, 1953-1962.

15. Lucas, X.; Grüning, B. A.; Bleher, S.; Günther, S. The Purchasable Chemical Space: A Detailed Picture. J. Chem. Inf. Model. 2015, 55, 915-924.

16. Feher, M.; Schmidt, J. M. Property Distributions: Differences between Drugs, Natural Products, and Molecules from Combinatorial Chemistry. J. Chem. Inf. Comput. Sci. 2003, 43, 218-227.

17. Henkel, T.; Brunne, R. M.; Müller, H.; Reichel, F. Statistical Investigation into the Structural Complementarity of Natural Products and Synthetic Compounds. Angew. Chem. Int. Ed. 1999, 38, 643-647.

18. Lee, M. L.; Schneider, G. Scaffold architecture and pharmacophoric properties of natural products and trade drugs: application in the design of natural product-based combinatorial libraries. J Comb Chem 2001, 3, 284-289.

19. Yongye, A. B.; Waddell, J.; Medina-Franco, J. L. Molecular Scaffold Analysis of Natural Products Databases in the Public Domain. Chem. Biol. Drug. Des. 2012, 80, 717-724.

20. Chen, Y.; Garcia de Lomana, M.; Friedrich, N.-O.; Kirchmair, J. Characterization of the Chemical Space of Known and Readily Obtainable Natural Products. J. Chem. Inf. Model. 2018, 58, 1518-1532.

21. Stahura, F. L.; Godden Jw Fau - Xue, L.; Xue L Fau - Bajorath, J.; Bajorath, J. Distinguishing between natural products and synthetic molecules by descriptor Shannon entropy analysis and binary QSAR calculations. J. Chem. Inf. Comput. Sci. 2000 40, 1245-1252.

22. Ertl, P.; Roggo, S.; Schuffenhauer, A. Natural Product-likeness Score and Its Application for Prioritization of Compound Libraries. J. Chem. Inf. Model. 2008, 48, 68-74.

23. Vanii Jayaseelan, K.; Moreno, P.; Truszkowski, A.; Ertl, P.; Steinbeck, C. Natural product-likeness score revisited: an open-source, open-data implementation. BMC Bioinformatics 2012, 13, 106.

24. Lachance, H.; Wetzel S Fau - Kumar, K.; Kumar K Fau - Waldmann, H.; Waldmann, H. Charting, navigating, and populating natural product chemical space for drug discovery. J. Med. Chem. 2012, 55, 5989-6001.

25. Fernanda, I. S.-G.; Pilón-Jiménez, B. A.; José, L. M.-F. Chemical space of naturally occurring compounds. Physical Sciences Reviews 2018, 4, 20180103.

26. Saldívar-González, F. I.; Lenci, E.; Trabocchi, A.; Medina-Franco, J. L. Exploring the chemical space and the bioactivity profile of lactams: a chemoinformatic study. RSC Advances 2019, 9, 27105-27116.

27. Ertl, P.; Schuhmann, T. Cheminformatics Analysis of Natural Product Scaffolds: Comparison of Scaffolds Produced by Animals, Plants, Fungi and Bacteria. Mol. Inform. 2020, 39, 2000017.

28. Grabowski, K.; Baringhaus Kh Fau - Schneider, G.; Schneider, G. Scaffold diversity of natural products: inspiration for combinatorial library design. Nat. Prod. Rep. 2008, 25, 892-904.

29. Miyao, T.; Reker, D.; Schneider, P.; Funatsu, K.; Schneider, G. Chemography of natural product space. Planta Med. 2015, 81, 429-435.

30. van Santen, J. A.; Jacob, G.; Singh, A. L.; Aniebok, V.; Balunas, M. J.; Bunsko, D.; Neto, F. C.; CastañoEspriu, L.; Chang, C.; Clark, T. N.; Cleary Little, J. L.; Delgadillo, D. A.; Dorrestein, P. C.; Duncan, K. R.; Egan, J. M.; Galey, M. M.; Haeckl, F. P. J.; Hua, A.; Hughes, A. H.; Iskakova, D.; Khadilkar, A.; Lee, J.-H.; Lee, S.; LeGrow, N.; Liu, D. Y.; Macho, J. M.; McCaughey, C. S.; Medema, M. H.; Neupane, R. P.; O’Donnell, T. J.; Paula, J. S.; Sanchez, L. M.; Shaikh, A. F.; Soldatou, S.; Terlouw, B. R.; Tran, T. A.; Valentine, M.; van der Hooft, J. J. J.; Vo, D. A.; Wang, M.; Wilson, D.; Zink, K. E.; Linington, R. G. The Natural Products Atlas: An Open Access Knowledge Base for Microbial Natural Products Discovery. ACS Central Science 2019, 5, 1824-1833. 
31. Díaz-Eufracio, B. I.; Palomino-Hernández, O.; Arredondo-Sánchez, A.; Medina-Franco, J. L. D-Peptide Builder: A Web Service to Enumerate, Analyze, and Visualize the Chemical Space of Combinatorial Peptide Libraries. Mol. Inform. 2020, 39, 2000035.

32. Sorokina, M.; Merseburger, P.; Rajan, K.; Yirik, M. A.; Steinbeck, C. Coconut Online: Collection of Open

Natural Products Database. Preprint 2019, 10.21203/rs.3.rs-75600/v1.

33. Irwin, J. J.; Tang, K. G.; Young, J.; Dandarchuluun, C.; Wong, B. R.; Khurelbaatar, M.; Moroz, Y. S.;

Mayfield, J.; Sayle, R. A. ZINC20-A Free Ultralarge-Scale Chemical Database for Ligand Discovery. J. Chem. Inf. Model. 2020.

34. Bishop, C. M.; Svensén, M.; Williams, C. K. I. GTM: The Generative Topographic Mapping. Neural Comput. 1998, 10, 215-234.

35. Lin, A.; Horvath, D.; Afonina, V.; Marcou, G.; Reymond, J.-L.; Varnek, A. Mapping of the Available Chemical Space versus the Chemical Universe of Lead-Like Compounds. ChemMedChem 2018, 13, 540-554.

36. Lin, A.; Beck, B.; Horvath, D.; Marcou, G.; Varnek, A. Diversifying chemical libraries with generative topographic mapping. J. Comput. Aided Mol. Des. 2019.

37. Zabolotna, Y.; Lin, A.; Horvath, D.; Marcou, G.; Volochnyuk, D. M.; Varnek, A. Chemography: Searching for Hidden Treasures. J. Chem. Inf. Model. 2020.

38. Grigalunas, M.; Burhop, A.; Christoforow, A.; Waldmann, H. Pseudo-natural products and natural productinspired methods in chemical biology and drug discovery. Curr. Opin. Chem. Biol. 2020, 56, 111-118.

39. ChemAxon. JChem, Version 20.8.3, ChemAxon, Ltd: Budapest, Hungary 2020.

40. Ruggiu, F.; Marcou, G.; Varnek, A.; Horvath, D. ISIDA Property-Labelled Fragment Descriptors. Mol. Inform. 2010, 29, 855-868.

41. Mendez, D.; Gaulton, A.; Bento, A. P.; Chambers, J.; De Veij, M.; Félix, E.; Magariños, M. P.; Mosquera, J. F.; Mutowo, P.; Nowotka, M.; Gordillo-Marañón, M.; Hunter, F.; Junco, L.; Mugumbate, G.; Rodriguez-Lopez, M.; Atkinson, F.; Bosc, N.; Radoux, C. J.; Segura-Cabrera, A.; Hersey, A.; Leach, A. R. ChEMBL: towards direct deposition of bioassay data. Nucleic Acids Res. 2019, 47, D930-D940.

42. Kohonen, T. Self-organized formation of topologically correct feature maps. Biol. Cybern. 1982, 43, 59-69.

43. Kireeva, N.; Baskin, I. I.; Gaspar, H. A.; Horvath, D.; Marcou, G.; Varnek, A. Generative Topographic Mapping (GTM): Universal Tool for Data Visualization, Structure-Activity Modeling and Dataset Comparison. Mol. Inform. 2012, 31, 301-312.

44. Gaspar, H. A.; Baskin, I. I.; Marcou, G.; Horvath, D.; Varnek, A. GTM-Based QSAR Models and Their Applicability Domains. Mol. Inform. 2015, 34, 348-356.

45. Lin, A.; Horvath, D.; Marcou, G.; Beck, B.; Varnek, A. Multi-task generative topographic mapping in virtual screening. J. Comput. Aided Mol. Des. 2019, 33, 331-343.

46. Horvath, D.; Marcou, G.; Varnek, A. Generative topographic mapping in drug design. Drug Discovery Today: Technologies 2020.

47. Sidorov, P.; Gaspar, H.; Marcou, G.; Varnek, A.; Horvath, D. Mappability of drug-like space: towards a polypharmacologically competent map of drug-relevant compounds. J. Comput. Aided Mol. Des. 2015, 29, 10871108.

48. $\quad$ Casciuc, I.; Zabolotna, Y.; Horvath, D.; Marcou, G.; Bajorath, J.; Varnek, A. Virtual Screening with Generative Topographic Maps: How Many Maps Are Required? J. Chem. Inf. Model. 2019, 59, 564-572.

49. Horvath, D.; Brown, J. B.; Marcou, G.; Varnek, A. An Evolutionary Optimizer of libsvm Models. Challenges 2014, 5, 450-472.

50. Tino, P.; Nabney, I. Hierarchical GTM: constructing localized nonlinear projection manifolds in a principled way. IEEE PAMI 2002, 24, 639-656.

51. Bickerton, G. R.; Paolini, G. V.; Besnard, J.; Muresan, S.; Hopkins, A. L. Quantifying the chemical beauty of drugs. Nat. Chem. 2012, 4, 90-98.

52. $\quad$ Patterson, D. E.; Cramer, R. D.; Ferguson, A. M.; Clark, R. D.; Weinberger, L. E. Neighborhood Behavior: A Useful Concept for Validation of "Molecular Diversity" Descriptors. J. Med. Chem. 1996, 39, 3049-3059. 